\title{
Multiple Fractional Dual Solutions in PEMC Backed Chiral Nihility Slab
}

\author{
Kanwer Aftab, ${ }^{1,2}$ M. Naveed, ${ }^{1,2}$ Aqeel A. Syed, ${ }^{1,2}$ \\ Shakeel Ahmed, ${ }^{1,2}$ and Q. A. Naqvi', 2 \\ ${ }^{1}$ Department of Electronics, Quaid-i-Azam University, Islamabad 45320, Pakistan \\ ${ }^{2}$ National University of Science and Technology (NUST), Islamabad 44000, Pakistan \\ Correspondence should be addressed to Q. A. Naqvi, nqaisar@yahoo.com
}

Received 18 May 2011; Accepted 22 August 2011

Academic Editor: Kue-Hong Chen

Copyright (C) 2011 Kanwer Aftab et al. This is an open access article distributed under the Creative Commons Attribution License, which permits unrestricted use, distribution, and reproduction in any medium, provided the original work is properly cited.

Inside a chiral nihility slab which is backed by perfect electromagnetic conductor, all valid fractional dual solutions are determined using the fractional curl operator. Fractional dual solutions may be regarded as intermediate step between the original and dual to the original solution. Corresponding fields outside the nihility slab for each case are also determined and analyzed. It is noted that only for one out of three cases the corresponding field outside chiral nihility slab is also fractional dual, while for other two cases, corresponding fields are not fractional dual. It is noted that time average power density vanishes inside the PEC-backed chiral nihility slab, while it exists outside the nihility slab.

\section{Introduction}

Chiral nihility is a special kind of chiral medium, for which the real part of permittivity and permeability are simultaneously zero or refractive index become zero at certain frequency known as nihility frequency. The realization of the chiral nihility medium has been discussed in [1-3]. Tretyakov et al. [1], showed that this material can be realized in a similar way as mixture with "ordinary" nihility by addig chiral inclusions all of the same handedness. At the frequency where the real parts of both permittivity and permeability become zeros, the chirality parameter is nonzero, and, the imaginary parts of all the parameters can be rather small compared to the chirality parameter. It has been proved that at the frequency equal to $6.38 \mathrm{GHz}$, both the permittivity and permeability of the material have zero real parts as discussed by Tretyakov et al. [2]. Also, Cheng et al. [3] proposed the realization of chiral nihility medium inside waveguide at frequency $0.5 \mathrm{GHz}$. Chiral nihility is extension of 
nihility concept, introduced by Lakhtakia [4] and studied by researchers [5-7], for ordinary dielectric medium. In chiral nihility, the two nihility, the two eigenwaves are still circularly polarized but one of them is a backward wave. For backward waves, phase velocity is antiparallel to the corresponding Poynting vector. Phenomena of negative refraction occurs when a plane wave enters from vacuum to chiral nihility medium.

Due to its interesting and extraordinary characteristics, chiral nihility has attracted many researchers [8-21]. Constitutive relations for isotropic chiral nihility medium are [2]

$$
\begin{gathered}
\mathbf{D}=-i \kappa \sqrt{\epsilon_{0} \mu_{0}} \mathbf{H}, \\
\mathbf{B}=i \kappa \sqrt{\epsilon_{0} \mu_{0}} \mathbf{E} .
\end{gathered}
$$

Taking time dependence as $\exp (i \omega t)$, Maxwell equations for chiral nihility medium can be written as

$$
\begin{gathered}
\nabla \times \mathbf{E}=k_{0} \kappa \mathbf{E}, \\
\nabla \times \mathbf{H}=k_{0} \kappa \mathbf{H},
\end{gathered}
$$

where $k_{0}=\omega / c$ is the wavenumber in vacuum. It must be noted that electric and magnetic fields in chiral nihility medium are not independent but are related through wave impedance

$$
\eta=\eta_{0} \lim _{\epsilon \rightarrow 0, \mu \rightarrow 0} \sqrt{\frac{\mu}{\epsilon}}
$$

It may be noted that, in the present paper, impedance of chiral nihility medium has been considered close to impedance of free space $\eta_{0}$.

According to the duality principle for ordinary isotropic medium, if $\left(\mathbf{E}, \eta_{0} \mathbf{H}\right)$ is one solution to Maxwell equations, then $\left(\eta_{0} \mathbf{H},-\mathbf{E}\right)$ is another solution to Maxwell equations. Solution $\left(\eta_{0} \mathbf{H},-\mathbf{E}\right)$ is termed as dual solution to the original solution $\left(\mathbf{E}, \eta_{0} \mathbf{H}\right)$, because Maxwell equations remain unchanged for both solutions. It is obvious, from above given Maxwell equations for chiral nihility medium, that if $(\mathbf{E}, \eta \mathbf{H})$ is one solution to Maxwell equations, then other solutions for which Maxwell equations remain unchanged are $(\mp \eta \mathbf{H}, \pm \mathbf{E})$, $(-\mathbf{E},-\eta \mathbf{H})$, and $( \pm \eta \mathbf{H}, \pm \mathbf{E})$. Set of solutions which may be discerned as dual solutions to the original solution $(\mathbf{E}, \eta \mathbf{H})$ is $\{(\eta \mathbf{H},-\mathbf{E}),(-\eta \mathbf{H}, \mathbf{E}),(-\mathbf{E},-\eta \mathbf{H})\}$, as discussed by Naqvi [11]. This means that simultaneous rotation of both electric and magnetic fields in clockwise or counterclockwise direction by amount of integer multiple of $\pi / 2$ is allowed to get the dual solution. Unallowed dual solution means that one field of original solution is being rotated in clockwise direction, while the other is being rotated counterclockwise and vice versa. That is, $(\eta \mathbf{H}, E)$ and $(-\eta \mathbf{H},-E)$ are invalid dual solutions. Now, if an achiral-chiral-nihility interface is excited by a uniform plane wave, there exists more than one dual to original solution in chiral nihility medium while only one dual to original solution in ordinary medium.

For an isotropic and homogeneous medium, the solutions which may be regarded as intermediate step between the original and dual to the original solutions may be obtained 
using the following relations:

$$
\begin{gathered}
\mathbf{E}_{\mathrm{fd}}=\frac{1}{\left(i k_{0}\right)^{\alpha}}(\nabla \times)^{\alpha} \mathbf{E}, \\
\eta_{0} \mathbf{H}_{\mathrm{fd}}=\frac{1}{\left(i k_{0}\right)^{\alpha}}(\nabla \times)^{\alpha} \eta_{0} \mathbf{H},
\end{gathered}
$$

where $\alpha$ is fractional parameter and $(\nabla \times)^{\alpha}$ means fractional curl operator. $k_{0}=\omega \sqrt{\mu_{0} \epsilon_{0}}$ is the wavenumber and $\eta_{0}=\sqrt{\mu_{0} / \epsilon_{0}}$ is impedance of the medium. The intermediate solutions between the above two sets of solutions may be obtained by varying parameter $\alpha$ between zero and one. A number of contributions in this direction are available in the published literature [22-40].

The other important material which is to be discussed here in this paper is the perfect electromagnetic (PEMC) material. PEMC material is the generalization of PEC and PMC materials. Perfect electromagnetic conductor (PEMC) was introduced by Lindell and Sihvola [41]. PEMC has attracted many researchers to contribute in the field of the field of electromagnetics using this material [42-54].

In this paper, we have determined all valid fractional dual solutions inside a chiral nihility slab which is backed by PEMC surface, using the fractional curl operator. Fractional dual solutions inside the PEMC-backed chiral nihility slab and corresponding fields for region outside the slab are determined and analyzed. It is noted that only for one out of three cases corresponding field outside chiral nihility slab is also fractional dual, while for other two cases, corresponding fields are not fractional dual. It is shown that time average power density vanishes inside the PEC-backed chiral nihility slab while it exists outside the nihility slab. It is seen that duality theorem provides a way to obtain solution of a problem without going into analytical details. In ordinary medium, only one solution/problem (known as dual) can be treated if original solution is known, whereas chirality nihility provides opportunity to address more than one such situations. Present study incorporates all possible (three possible dual solutions) combinations of original and dual to original solutions.

\section{Chiral Nihility Slab Backed by PEMC}

Consider a slab of chiral nihility metamaterial. The slab is of infinite length and is backed by PEMC. Front face of the chiral nihility slab is located at $z=d_{1}$, while perfect electromagnetic conductor is located at location $z=d_{2}$, where $d_{2}>d_{1}$.

A linearly polarized uniform plane wave, with time dependency time harmonic $\exp (-j \omega t)$, is incident on the chiral nihility slab backed by PEMC. For the sake of simplicity, it is assumed that direction of incidence is parallel to the normal at chiral nihility slab. The electric and magnetic fields inside and outside the grounded chiral nihility slab may be written in terms of unknown coefficients as

$$
\begin{aligned}
\mathbf{E}_{0}= & \widehat{\mathbf{x}} \exp \left(i k_{0 z} z\right)+A^{-} \mathbf{N}_{R}^{-} \exp \left(-i k_{0 z} z\right)+B^{-} \mathbf{N}_{L}^{-} \exp \left(-i k_{0 z} z\right), \quad z<d_{1}, \\
\mathbf{E}_{1}= & E^{+} \mathbf{M}_{R}^{+} \exp \left(i k_{z}^{+} z\right)+F^{+} \mathbf{M}_{L}^{+} \exp \left(i k_{z}^{-} z\right) \\
& +E^{-} \mathbf{M}_{R}^{-} \exp \left(-i k_{z}^{+} z\right)+F^{-} \mathbf{M}_{L}^{-} \exp \left(-i k_{z}^{-} z\right), \quad d_{1}<z<d_{2},
\end{aligned}
$$




$$
\begin{aligned}
& \mathbf{H}_{0}=\frac{1}{\eta_{0}} \widehat{\mathbf{y}} \exp \left(i k_{0 z} z\right)-\frac{i}{\eta_{0}}\left\{A^{-} \mathbf{N}_{R}^{-} \exp \left(-i k_{0 z} z\right)-B^{-} \mathbf{N}_{L}^{-} \exp \left(-i k_{0 z} z\right)\right\}, z<d_{1}, \\
& H_{1}=\frac{-i}{\eta}\left[E^{+} \mathbf{M}_{R}^{+} \exp \left(i k_{z}^{+} z\right)-F^{+} \mathbf{M}_{L}^{+} \exp \left(i k_{z}^{-} z\right)+E^{-} \mathbf{M}_{R}^{-} \exp \left(-i k_{z}^{+} z\right)-F^{-} \mathbf{M}_{L}^{-} \exp \left(-i k_{z}^{-} z\right)\right], \\
& d_{1}<z<d_{2},
\end{aligned}
$$

where

$$
\begin{aligned}
& \mathbf{N}_{R}^{ \pm}=\widehat{x} \pm i \widehat{y}, \\
& \mathbf{N}_{L}^{ \pm}=\widehat{x} \mp i \widehat{y}, \\
& \mathbf{M}_{R}^{ \pm}=\widehat{x} \pm i \widehat{y}, \\
& \mathbf{M}_{L}^{ \pm}=\widehat{x} \mp i \widehat{y} .
\end{aligned}
$$

Superscript \pm in (2.2) represents the eigenwaves propagating in the $\pm z$ direction. The subscript $R$ and $L$ refer to the RCP and LCP eigenwaves satisfying the dispersion relations

$$
k_{z}^{ \pm}= \pm \omega \kappa,
$$

at the nihility frequency. In the above equations, $k_{0 z}=\omega \sqrt{\mu_{0} \epsilon_{0}}$ and $\eta=\sqrt{\mu / \epsilon}$. It may be noted that relation $k_{z}^{+}=-k_{z}^{-}$holds for all modes propagating inside the slab.

Unknown coefficients in field expressions (2.1) may be obtained by using the related boundary conditions. At $z=d_{2}$, tangential components of field quantity $\left(M \mathrm{E}_{1}+\mathbf{H}_{1}\right)$ must be [40].Tangential components of electric and magnetic fields across the dielectric interface located at $z=d_{1}$ must be continuous. Application of these boundary conditions yield unknown coefficients

$$
\begin{gathered}
E^{-}=\frac{1}{2}\left(\frac{M \eta+i}{i-M \eta}\right) \exp \left(i k_{0 z} d_{1}+i k_{z}^{+} d_{1}\right), \\
E^{+}=\frac{\exp \left(i k_{0 z} d_{1}-i k_{z}^{+} d_{1}\right)}{2}, \\
A^{-}=\left(\frac{M \eta+i}{i-M \eta}\right) \frac{\exp \left(2 i k_{0 z} d_{1}\right)}{2}, \\
B^{-}=\left(\frac{i-M \eta}{M \eta+i}\right) \frac{\exp \left(2 i k_{0 z} d_{1}\right)}{2} .
\end{gathered}
$$

It may be noted that all coefficients are independent of parameter $d_{2}$.

\section{Fractional Dual Solutions in Chiral Nihility Slab}

Using the concept of fractional curl operator $(\nabla \times)^{\alpha}[14]$, we can find fractional dual solutions to the Maxwell equations in chiral nihility slab. Discussion has been divided into three cases as: 
Case 1. Fractional dual fields between $(\mathbf{E}, \eta \mathbf{H})$ and $(\eta \mathbf{H},-\mathbf{E})$ for region inside and outside the slab may be obtained from Maxwell equations as

$$
\begin{aligned}
\mathbf{E}_{0 \mathrm{fd}}^{ \pm} & =\left(\widehat{\mathbf{k}}_{i} \times\right)^{\alpha} \mathbf{E}_{0}^{ \pm}, \\
\eta_{0} \mathbf{H}_{0 \mathrm{fd}}^{ \pm} & =\left(\widehat{\mathbf{k}}_{i} \times\right)^{\alpha} \eta_{0} \mathbf{H}_{0}^{ \pm}, \\
\mathbf{E}_{1 \mathrm{fd}}^{ \pm} & =\left(\widehat{\mathbf{k}}_{i} \times\right)^{\alpha} \mathbf{E}_{1}^{ \pm}, \\
\eta \mathbf{H}_{1 \mathrm{fd}} & =\left(\widehat{\mathbf{k}}_{i} \times\right)^{\alpha} \eta \mathbf{H}_{1}^{ \pm} .
\end{aligned}
$$

Above fields must satisfy the Maxwell equations. Subscript fd stands for fractional dual. Sign \pm are for propagation in positive and negative $z$-direction. In order to deal with above equations, eigenvalues and eigenvector of operators $\left(\widehat{\mathbf{k}}_{i} \times\right)= \pm \widehat{z} \times$ are required. So, the eigenvalues and eigenvectors of these cross-product operators are given below. Eigenvalues and eigenvectors of operator $\widehat{\mathbf{k}}_{1} \times=\widehat{z} \times$

$$
\begin{gathered}
\mathbf{A}_{1}=\frac{1}{\sqrt{2}}[\widehat{x}+i \widehat{y}], \quad a_{1}=-i, \\
\mathbf{A}_{2}=\frac{1}{\sqrt{2}}[\widehat{x}-i \widehat{y}], \quad a_{2}=+i, \\
\mathbf{A}_{3}=i \widehat{z}, \quad a_{3}=0 .
\end{gathered}
$$

Eigenvalues and eigenvectors of operator $\widehat{\mathbf{k}}_{2} \times=-\widehat{z} \times$ are

$$
\begin{gathered}
\mathbf{A}_{1}=\frac{1}{\sqrt{2}}[\widehat{x}+i \hat{y}], \quad a_{1}=+i, \\
\mathbf{A}_{2}=\frac{1}{\sqrt{2}}[\widehat{x}-i \hat{y}], \quad a_{2}=-i, \\
\mathbf{A}_{3}=-i \widehat{z}, \quad a_{3}=0 .
\end{gathered}
$$

Fractional dual fields are obtained by fractionalizing the eigenvalues of corresponding linear operator; that is,

$$
\begin{aligned}
\mathbf{E}_{0 \mathrm{fd}}= & {\left[(-i)^{\alpha} \frac{1}{\sqrt{2}} \mathbf{A}_{1}+(+i)^{\alpha} \frac{1}{\sqrt{2}} \mathbf{A}_{2}\right] \exp \left(i k_{0 z} z\right) } \\
& +\left[(-i)^{\alpha} A^{-} \mathbf{N}_{R}^{-}+(i)^{\alpha} B^{-} \mathbf{N}_{L}^{-}\right] \exp \left(-i k_{0 z} z\right), \\
\mathbf{E}_{1 \mathrm{fd}}= & {\left[(-i)^{\alpha} E^{+} \mathbf{M}_{R}^{+} \exp \left(i k_{z}^{+} z\right)+(i)^{\alpha} F^{+} \mathbf{M}_{L}^{+} \exp \left(i k_{z}^{-} z\right)+(-i)^{\alpha} E^{-} \mathbf{M}_{R}^{-} \exp \left(-i k_{z}^{+} z\right)\right.} \\
& \left.+(i)^{\alpha} F^{-} \mathbf{M}_{L}^{-} \exp \left(-i k_{z}^{-} z\right)\right],
\end{aligned}
$$




$$
\begin{aligned}
\eta_{0} \mathbf{H}_{0 \mathrm{fd}}= & {\left[(-i)^{\alpha+1} \frac{1}{\sqrt{2}} \mathbf{A}_{1}+(+i)^{\alpha+1} \frac{1}{\sqrt{2}} \mathbf{A}_{2}\right] \exp \left(i k_{0 z} z\right) } \\
& +\left[(-i)^{\alpha+1} A^{-} \mathbf{N}_{R}^{-}+(i)^{\alpha+1} B^{-} \mathbf{N}_{L}^{-}\right] \exp \left(-i k_{0 z} z\right), \\
\eta \mathbf{H}_{1 \mathrm{fd}}= & {\left[(-i)^{\alpha+1} E^{+} \mathbf{M}_{R}^{+} \exp \left(i k_{z}^{+} z\right)+(i)^{\alpha+1} F^{+} \mathbf{M}_{L}^{+} \exp \left(i k_{z}^{-} z\right)+(-i)^{\alpha+1} E^{-} \mathbf{M}_{R}^{-} \exp \left(-i k_{z}^{+} z\right)\right.} \\
& \left.+(i)^{\alpha+1} F^{-} \mathbf{M}_{L}^{-} \exp \left(-i k_{z}^{-} z\right)\right] .
\end{aligned}
$$

Expressing $k_{z}^{-}$as $\left(-k_{z}^{+}\right)$, above equations yields the following:

$$
\begin{aligned}
& \mathbf{E}_{0 f d}=\left[\cos \left(\frac{\alpha \pi}{2}\right) \hat{x}+\sin \left(\frac{\alpha \pi}{2}\right) \hat{y}\right] \exp \left(i k_{0 z} z\right) \\
& -\frac{1}{(M \eta)^{2}+1}\left[\left\{\left((M \eta)^{2}-1\right) \cos \left(\frac{\alpha \pi}{2}\right)+2 M \eta \sin \left(\frac{\alpha \pi}{2}\right)\right\} \widehat{x}\right. \\
& \left.-\left\{\left((M \eta)^{2}-1\right) \sin \left(\frac{\alpha \pi}{2}\right)-2 M \eta \cos \left(\frac{\alpha \pi}{2}\right)\right\} \hat{y}\right] \exp \left(-i k_{0 z}\left(z-2 d_{1}\right)\right), \\
& \mathbf{E}_{1 f d}=\frac{(-i \widehat{x}+\hat{y})}{M \eta+i}\left[M \eta \sin \left(\frac{\alpha \pi}{2}\right)-\cos \left(\frac{\alpha \pi}{2}\right)\right] \exp \left(i k_{0 z} d_{1}+i k_{z}^{+}\left(z-d_{1}\right)\right) \\
& +\frac{(-i \hat{x}-\widehat{y})}{M \eta+i}\left[M \eta \sin \left(\frac{\alpha \pi}{2}\right)-\cos \left(\frac{\alpha \pi}{2}\right)\right]\left[\frac{M \eta+i}{i-M \eta}\right] \times \exp \left(i k_{0 z} d_{1}-i k_{z}^{+}\left(z-d_{1}\right)\right), \\
& \eta_{0} \mathbf{H}_{0 f d}=\left[\cos \left(\frac{(\alpha+1) \pi}{2}\right) \hat{x}+\sin \left(\frac{(\alpha+1) \pi}{2}\right) \hat{y}\right] \exp \left(i k_{0 z} z\right) \\
& -\frac{1}{(M \eta)^{2}+1}\left[\left\{\left((M \eta)^{2}-1\right) \cos \left(\frac{\alpha \pi}{2}\right)+2 M \eta \sin \left(\frac{(\alpha+1) \pi}{2}\right)\right\} \widehat{x}\right. \\
& \left.-\left\{\left((M \eta)^{2}-1\right) \sin \left(\frac{(\alpha+1) \pi}{2}\right)-2 M \eta \cos \left(\frac{(\alpha+1) \pi}{2}\right)\right\} \widehat{y}\right] \\
& \times \exp \left(-i k_{0 z}\left(z-2 d_{1}\right)\right) \text {, } \\
& \eta \mathbf{H}_{1 f d}=\frac{(-i \widehat{x}+\widehat{y})}{M \eta+i}\left[M \eta \sin \left(\frac{(\alpha+1) \pi}{2}\right)-\cos \left(\frac{(\alpha+1) \pi}{2}\right)\right] \times \exp \left(i k_{0 z} d_{1}+i k_{z}^{+}\left(z-d_{1}\right)\right) \\
& +\frac{(-i \widehat{x}-\widehat{y})}{M \eta+i}\left[M \eta \sin \left(\frac{(\alpha+1) \pi}{2}\right)-\cos \left(\frac{(\alpha+1) \pi}{2}\right)\right]\left[\frac{M \eta+i}{i-M \eta}\right] \\
& \times \exp \left(i k_{0 z} d_{1}-i k_{z}^{+}\left(z-d_{1}\right)\right) \text {. }
\end{aligned}
$$

Boundary conditions can be easily verified at two interfaces. Changing values of $\alpha$ between zero and one, one can find behavior of fields inside intermediate geometries. For $\alpha=0$ and $\alpha=1$, one reproduces the original and dual to original solutions in chiral nihility slab. 
It may be noted that corresponding field outside the slab is also fractional dual between the original and dual to the original field.

Case 2. Inside the chiral nihility slab, solutions which may be regarded as intermediate step between the original solution $(\mathbf{E}, \eta \mathbf{H})$ and dual to original solution $(-\eta \mathbf{H}, \mathbf{E})$ are obtained as

$$
\begin{aligned}
\mathbf{E}_{1 \mathrm{fd}}= & \frac{-\widehat{y}+i \widehat{x}}{M \eta+1}\left[M \eta \sin \left(\frac{\alpha \pi}{2}\right)+\cos \left(\frac{\alpha \pi}{2}\right)\right] \exp \left(i k_{0 z} d_{1}+i k_{z}^{+}\left(z-d_{1}\right)\right) \\
& +\frac{\widehat{y}+i \widehat{x}}{i-M \eta}\left[M \eta \sin \left(\frac{\alpha \pi}{2}\right)+\cos \left(\frac{\alpha \pi}{2}\right)\right] \exp \left(i k_{0 z} d_{1}-i k_{z}^{+}\left(z+d_{1}\right)\right), \\
\eta_{0} \mathbf{H}_{1 \mathrm{fd}}= & (\widehat{y}-i \widehat{x}) \sin \left(\frac{(\alpha+1) \pi}{2}\right) \exp \left(i k_{0 z} d_{1}+i k_{z}^{+}\left(z-d_{1}\right)\right) \\
& -(\widehat{y}+i \widehat{x}) \frac{M \eta+i}{i-M \eta} \sin \left(\frac{(\alpha+1) \pi}{2}\right) \exp \left(i k_{0 z} d_{1}-i k_{z}^{+}\left(z-d_{1}\right)\right) .
\end{aligned}
$$

Corresponding fields outside the nihility slab is given below

$$
\begin{aligned}
\mathbf{E}_{0 \mathrm{fd}}= & {\left[\cos \left(\frac{\alpha \pi}{2}\right) \hat{x}-\sin \left(\frac{\alpha \pi}{2}\right) \hat{y}\right] \exp \left(i k_{0 z} z\right) } \\
& -\frac{1}{1+(M \eta)^{2}}\left[\left(\left((M \eta)^{2}-1\right) \cos \left(\frac{\alpha \pi}{2}\right)-2 M \eta \sin \left(\frac{\alpha \pi}{2}\right)\right) \widehat{x}\right. \\
& \left.+\left(\left((M \eta)^{2}-1\right) \sin \left(\frac{\alpha \pi}{2}\right)+2 M \eta \cos \left(\frac{\alpha \pi}{2}\right)\right) \hat{y}\right] \times \exp \left(-i k_{0 z}\left(z-2 d_{1}\right)\right), \\
\eta_{0} \mathbf{H}_{0 \mathrm{fd}}= & {\left[\cos \left(\frac{(\alpha+1) \pi}{2}\right) \hat{x}-\sin \left(\frac{(\alpha+1) \pi}{2}\right) \hat{y}\right] \exp \left(i k_{0 z} z\right)-\frac{1}{1+(M \eta)^{2}} } \\
& \times\left[\left(\left((M \eta)^{2}-1\right) \cos \left(\frac{(\alpha+1) \pi}{2}\right)-2 M \eta \sin \left(\frac{\alpha+1 \pi}{2}\right)\right) \widehat{x}+\left(\left((M \eta)^{2}-1\right)\right.\right. \\
& \left.\left.\quad \times \sin \left(\frac{(\alpha+1) \pi}{2}\right)+2 M \eta \cos \left(\frac{(\alpha+1) \pi}{2}\right)\right) \hat{y}\right] \times \exp \left(-i k_{0 z}\left(z-2 d_{1}\right)\right) .
\end{aligned}
$$

Equivalent cross-product operator for the corresponding field outside the nihility slab is $\left(\widehat{\mathbf{k}}_{i} \times\right)$ $=\mp \widehat{z} \times$. It may be noted that outside fields are not fractional dual solutions.

Case 3. Solutions which may be regarded as intermediate step between the original solution $(\mathbf{E}, \eta \mathbf{H})$ and $(-\mathbf{E},-\eta \mathbf{H})$ are obtained as

$$
\begin{aligned}
\mathbf{E}_{1 \mathrm{fd}}= & \frac{-\widehat{y}+i \widehat{x}}{M \eta+1}[\cos (\alpha \pi)-M \eta \sin (\alpha \pi)] \exp \left(i k_{0 z} d_{1}+i k_{z}^{+}\left(z-d_{1}\right)\right) \\
& +\frac{\widehat{y}+i \widehat{x}}{i-M \eta}[\cos (\alpha \pi)-M \eta \sin (\alpha \pi)] \exp \left(i k_{0 z} d_{1}-i k_{z}^{+}\left(z-d_{1}\right)\right)
\end{aligned}
$$




$$
\begin{aligned}
\eta_{0} \mathbf{H}_{1 \mathrm{fd}}= & (\widehat{y}-i \widehat{x}) \sin \left(\frac{(2 \alpha+1) \pi}{2}\right) \exp \left(i k_{0 z} d_{1}+i k_{z}^{+}\left(z-d_{1}\right)\right) \\
& -(\widehat{y}+i \widehat{x}) \frac{M \eta+i}{i-M \eta} \sin \left(\frac{(2 \alpha+1) \pi}{2}\right) \exp \left(i k_{0 z} d_{1}-i k_{z}^{+}\left(z-d_{1}\right)\right) .
\end{aligned}
$$

Corresponding fields outside the nihility slab are

$$
\begin{aligned}
\mathbf{E}_{0 \mathrm{fd}}= & {[\cos (\alpha \pi) \hat{x}+\sin (\alpha \pi) \hat{y}] \exp \left(i k_{0 z} z\right)-\frac{1}{1+(M \eta)^{2}}\left[\left((M \eta)^{2}-1\right) \cos (\alpha \pi)+2 M \eta \sin (\alpha \pi)\right] \widehat{x} } \\
& -\left[\left((M \eta)^{2}-1\right) \sin (\alpha \pi)-2 M \eta \cos \alpha \pi\right] \widehat{y} \exp \left(-i k_{0 z}\left(z-2 d_{1}\right)\right), \\
\eta_{0} \mathbf{H}_{0 \mathrm{fd}}= & {\left[\cos \left(\frac{(2 \alpha+1) \pi}{2}\right) \hat{x}+\sin \left(\frac{(2 \alpha+1) \pi}{2}\right) \hat{y}\right] \exp \left(i k_{0 z} z\right) } \\
& -\frac{1}{1+(M \eta)^{2}}\left[\left((M \eta)^{2}-1\right) \cos \left(\frac{(2 \alpha+1) \pi}{2}\right)+2 M \eta \cos \left(\frac{(2 \alpha+1) \pi}{2}\right)\right] \widehat{x} \\
& -\left[\left((M \eta)^{2}-1\right) \sin \left(\frac{(2 \alpha+1) \pi}{2}\right)-2 M \eta \sin \left(\frac{(2 \alpha+1) \pi}{2}\right)\right] \widehat{y} \times \exp \left(-i k_{0 z}\left(z-2 d_{1}\right)\right) .
\end{aligned}
$$

Equivalent cross-product operator for the corresponding field outside the nihility slab is $\left(\widehat{\mathbf{k}}_{i} \times\right)$ $= \pm i \widehat{z} \times$. It may be noted that outside fields do not construct fractional dual solution.

The average power density $P_{\mathrm{av}}(z)$ can be obtained using the relation

$$
P_{\mathrm{av}}(z)=\frac{1}{2} \mathfrak{R}\left(\mathbf{E}(z) \times \mathbf{H}^{*}(z)\right) .
$$

It is noted that average power density is zero inside the chiral nihility slab, whereas it has nonzero value outside the slab.

\section{Conclusions}

It is concluded that inside the chiral nihility slab, three dual to original solution are possible, while outside the chiral nihility slab, only one dual to original solution exist. Out of three dual solutions for region inside the chiral nihility slab, only one dual solution has correspondence with the dual solution for region outside the slab. Fractional dual solutions inside the PEMCbacked chiral nihility slab and corresponding fields for region outside the slab are determined and analyzed. Average power density outside the slab is nonzero, whereas it yields zero for region inside the slab.

\section{References}

[1] S. Tretyakov, I. Nefedov, A. Sihvola, and S. Maslovski, "A metamaterial with extreme properties: the chiral nihility," in Proceedings of the Progress in Electromagnetics Research Symposium, Honolulu, Hawaii, USA, 2003. 
[2] S. Tretyakov, I. Nefedov, A. Sihvola, S. Maslovski, and C. Simovski, "Waves and energy in chiral nihility," Journal of Electromagnetic Waves and Applications, vol. 17, no. 5, pp. 695-706, 2003.

[3] Q. Cheng, T. J. Cui, and C. Zhang, "Waves in planar waveguide containing chiral nihility metamaterial," Optics Communications, vol. 276, no. 2, pp. 317-321, 2007.

[4] A. Lakhtakia, "An electromagnetic trinity from negative permittivity and negative permeability," International Journal of Infrared and Millimeter Waves, vol. 22, no. 12, pp. 1731-1734, 2001.

[5] A. Lakhtakia and J. B. Geddes III, "Scattering by a nihility cylinder," International Journal of Electronics and Communications, vol. 61, no. 1, pp. 62-65, 2007.

[6] A. Lakhtakia, "Scattering by a nihility sphere," Microwave and Optical Technology Letters, vol. 48, no. 5, pp. 895-896, 2006.

[7] S. Ahmed and Q. A. Naqvi, "Scattering of electromagnetic waves by a coated nihility cylinder," Journal of Infrared, Millimeter, and Terahertz Waves, vol. 30, no. 10, pp. 1044-1052, 2009.

[8] C.-W. Qiu, N. Burokur, S. Zouhdi, and L. W. Li, “Chiral nihility effects on energy flow in chiral materials," Journal of Optical Society of America A, vol. 25, no. 1, pp. 55-63, 2008.

[9] C. Zhang and T. J. Cui, "Negative reflections of electromagnetic waves in a strong chiral medium," Applied Physics Letters, vol. 91, no. 19, Article ID 194101, 2007.

[10] Q. A. Naqvi, "Planar slab of chiral nihility metamaterial backed by fractional dual/PEMC interface," Progress in Electromagnetics Research, vol. 85, pp. 381-391, 2008.

[11] Q. A. Naqvi, "Fractional dual solutions to the Maxwell equations in chiral nihility medium," Optics Communications, vol. 282, no. 10, pp. 2016-2018, 2009.

[12] Q. A. Naqvi, "Fractional dual solutions in grounded chiral nihility slab and their effect on outside fields," Journal of Electromagnetic Waves and Applications, vol. 23, no. 5, pp. 773-784, 2009.

[13] Q. A. Naqvi, "Fractional dual interface in chiral nihility medium," Progress in Electromagnetics Research Letters, vol. 8, pp. 135-142, 2009.

[14] J. Dong and C. Xu, "Characteristics of guided modes in planar chiral nihility meta-material waveguides," Progress In Electromagnetics Research B, no. 14, pp. 107-126, 2009.

[15] J. Dong and C. Xu, "Surface polaritons in planar chiral nihility meta-material waveguides," Optics Communications, vol. 282, no. 19, pp. 3899-3904, 2009.

[16] N. Engheta, "Fractional curl operator in electromagnetics," Microwave and Optical Technology Letters, vol. 17, no. 2, pp. 86-91, 1998.

[17] V. R. Tuz and C. W. Qiu, "Semi-infinite chiral nihility photonics: parametric dependence, wave tunneling and rejection," Progress in Electromagnetics Research, vol. 103, pp. 139-152, 2010.

[18] C. W. Qiu, L. W. Li, H. Y. Yao, and S. Zouhdi, "Properties of Faraday chiral media: green dyadics and negative refraction," Physical Review B, vol. 74, no. 11, Article ID 115110, 2006.

[19] S. R. Qamar, A. Naqvi, A. A. Syed, and Q. A. Naqvi, "Radiation characteristics of elementary sources located in unbounded chiral nihility metamaterial," Journal of Electromagnetic Waves and Applications, vol. 25, no. 5-6, pp. 713-722, 2011.

[20] M. A. Baqir, A. A. Syed, and Q. A. Naqvi, "Electromagnetic fields in a circular waveguide containing chiral nihility metamaterial," Progress In Electromagnetics Research M, vol. 16, pp. 85-93, 2011.

[21] M. Taj, A. Naqvi, A. A. Syed, and Q. A. Naqvi, "Study of focusing of a cylindrical interface of chiral nihility-chiral nihility media using Maslov's method," Progress In Electromagnetics Research Letters, vol. 22, pp. 181-190, 2011.

[22] Q. A. Naqvi and A. A. Rizvi, "Fractional dual solutions and corresponding sources," Journal of Electromagnetic Waves and Applications, vol. 25, pp. 223-238, 2000.

[23] Q. A. Naqvi, G. Murtaza, and A. A. Rizvi, "Fractional dual solutions to Maxwell equations in homogeneous chiral media," Optics Communications, vol. 178, no. 1, pp. 27-30, 2000.

[24] A. Lakhtakia, "A representation theorem involving fractional derivatives for linear homogeneous chiral media," Microwave and Optical Technology Letters, vol. 28, no. 6, pp. 385-386, 2001.

[25] Q. A. Naqvi and M. Abbas, "Fractional duality and metamaterials with negative permittivity and permeability," Optics Communications, vol. 227, no. 1-3, pp. 143-146, 2003.

[26] Q. A. Naqvi and M. Abbas, "Complex and higher order fractional curl operator in electromagnetics," Optics Communications, vol. 241, no. 4-6, pp. 349-355, 2004.

[27] A. Hussain and Q. A. Naqvi, "Fractional curl operator in chiral medium and fractional non-symmetric transmission line," Progress in Electromagnetics Research, vol. 59, pp. 319-335, 2006.

[28] A. Hussain, S. Ishfaq, and Q. A. Naqvi, "Fractional curl operator and fractional waveguides," Progress in Electromagnetics Research, vol. 63, pp. 319-335, 2006.

[29] A. Hussain, M. Faryad, and Q. A. Naqvi, "Fractional curl operator and fractional Chiro-waveguide," Journal of Electromagnetic Waves and Applications, vol. 21, no. 8, pp. 1119-1129, 2007. 
[30] A. Hussain, Q. A. Naqvi, and M. Abbas, "Fractional duality and perfect electromagnetic conductor (PEMC)," Progress in Electromagnetics Research, vol. 71, pp. 85-94, 2007.

[31] A. Hussain and Q. A. Naqvi, "Perfect electromagnetic conductor (PEMC) and fractional waveguide," Progress in Electromagnetics Research, vol. 73, pp. 61-69, 2007.

[32] M. Faryad and Q. A. Naqvi, "Fractional rectangular waveguide," Progress in Electromagnetics Research, vol. 75, pp. 383-396, 2007.

[33] H. Maab and Q. A. Naqvi, "Fractional surface waveguide," Progress In Electromagnetics Research C, vol. 1, pp. 199-209, 2008.

[34] A. Hussain, M. Faryad, and Q. A. Naqvi, "Fractional waveguide with impedance walls," Progress In Electromagnetics Research C, vol. 4, pp. 191-204, 2008.

[35] H. Maab and Q. A. Naqvi, "Fractional rectangular cavity resonator," Progress in Electromagnetics Research B, vol. 9, pp. 69-82, 2008.

[36] M. Abbas, S. A. Naqvi, and Q. A. Naqvi, "Foster reactance theorem in fractional dual guiding structures," International Journal of Microwave and Optical Technology, vol. 3, no. 2, pp. 85-87, 2008.

[37] F. Mustafa, M. Faryad, and Q. A. Naqvi, "Fractional dual solutions using calculus of differential forms," Journal of Electromagnetic Waves and Applications, vol. 22, no. 2-3, pp. 399-410, 2008.

[38] S. A. Naqvi, M. Faryad, Q. A. Naqvi, and M. Abbas, "Fractional duality in homogeneous bi-isotropic medium," Progress in Electromagnetics Research, vol. 78, pp. 159-172, 2008.

[39] E. I. Veliev and N. Engheta, "Fractional curl operator in reflection problems," in Proceedings of the 10th International Conference on Mathematical Methods in Electromagnetic Theory, pp. 228-230, Ukraine, September 2004.

[40] M. V. Ivakhnychenko, E. I. Veliev, and T. M. Ahmedov, "Fractional operators approach in electromagnetic wave reflection problems," Journal of Electromagnetic Waves and Applications, vol. 21, no. 13, pp. 1787-1802, 2007.

[41] I. V. Lindell and A. H. Sihvola, "Perfect electromagnetic conductor," Journal of Electromagnetic Waves and Applications, vol. 19, no. 7, pp. 861-869, 2005.

[42] I. V. Lindell and A. H. Sihvola, "Realization of the PEMC boundary," IEEE Transactions on Antennas and Propagation, vol. 53, no. 9, pp. 3012-3018, 2005.

[43] S. Ahmed and Q. A. Naqvi, "Electromagnetic scattering from a perfect electromagnetic conductor cylinder buried in a dielectric half-space," Progress in Electromagnetics Research, vol. 78, pp. 25-38, 2008.

[44] S. Ahmed and Q. A. Naqvi, "Electromagnetic scattering from parallel perfect electromagnetic conductor cylinders of circular cross-sections using an iterative procedure," Journal of Electromagnetic Waves and Applications, vol. 22, no. 7, pp. 987-1003, 2008.

[45] S. Ahmed and Q. A. Naqvi, "Electromagnetic scattering from a two dimensional perfect electromagnetic conductor (PEMC) strip and PEMC strip grating simulated by circular cylinders," Optics Communications, vol. 281, no. 17, pp. 4211-4218, 2008.

[46] S. Ahmed and Q. A. Naqvi, "Electromagnetic scattering from a perfect electromagnetic conductor circular cylinder coated with a metamaterial having negative permittivity and/or permeability," Optics Communications, vol. 281, no. 23, pp. 5664-5670, 2008.

[47] S. Ahmed and Q. A. Naqvi, "Electromagnetic scattering of two or more incident plane waves by a perfect electromagnetic conductor cylinder coated with a metamaterial," Progress In Electromagnetics Research B, vol. 10, pp. 75-90, 2008.

[48] S. Ahmed and Q. A. Naqvi, "Directive em radiation of a line source in the presence of a coated nihility Cylinder," Journal of Electromagnetic Waves and Applications, vol. 23, no. 5, pp. 761-771, 2009.

[49] S. Ahmed and Q. A. Naqvi, "Directive em radiation of a line source in the presence of a coated PEMC circular cylinder," Progress in Electromagnetics Research, vol. 92, pp. 91-102, 2009.

[50] A. Shehzad, S. Ahmed, and Q. A. Naqvi, "Electromagnetic scattering from a coated PEMC cylinder buried in a dielectric half space," Optics Communications, vol. 283, pp. 4563-4571, 2010.

[51] S. Ahmed and Q. A. Naqvi, "Electromagnetic scattering from a chiral-coated nihility cylinder," Progress In Electromagnetics Research Letters, vol. 18, pp. 41-50, 2010.

[52] A. Naqvi, S. Ahmed, and Q. A. Naqvi, "Perfect electromagnetic conductor and fractional dual interface placed in a chiral nihility medium," Journal of Electromagnetic Waves and Applications, vol. 24, no. 14-15, pp. 1991-1999, 2010. 
[53] M. Naveed, S. Ahmed, and Q. A. Naqvi, "Scattering of electromagnetic plane waves from a coated PEMC circular cylinder placed under PEC wide double wedge," Mathematical Problems in Engineering, vol. 2010, Article ID 254025, 26 pages, 2010.

[54] S. Ahmed, M. Naveed, A. Ghaffar, M. A. Abbas, and Q. A. Naqvi, "Electromagnetic scattering from parallel nihility cylinders coated by dissipative and dispersive DNG materials," Mathematical Problems in Engineering, vol. 2011, Article ID 27605, 12 pages, 2011. 


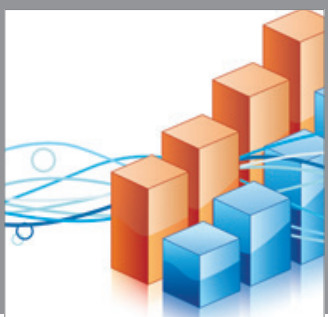

Advances in

Operations Research

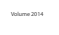

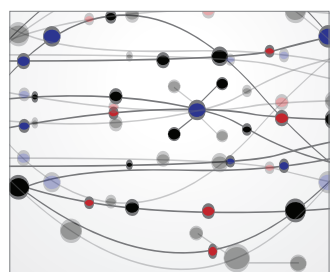

\section{The Scientific} World Journal
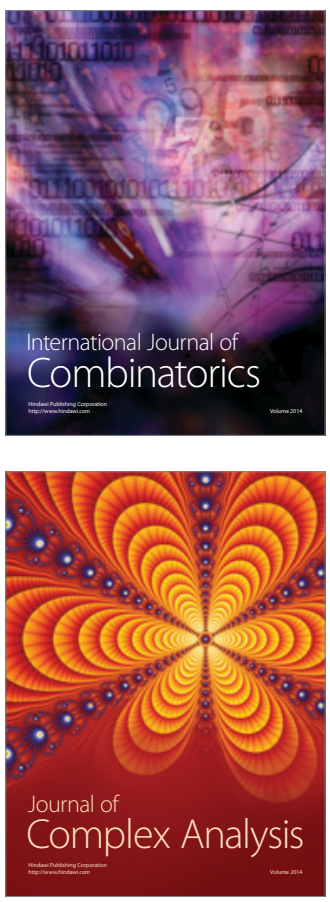

International Journal of

Mathematics and

Mathematical

Sciences
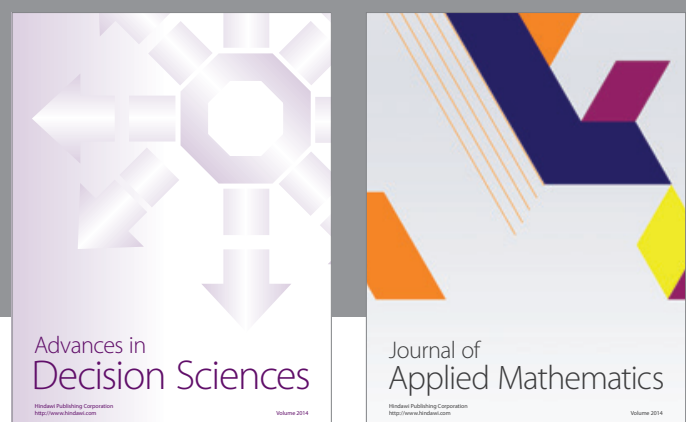

Journal of

Applied Mathematics
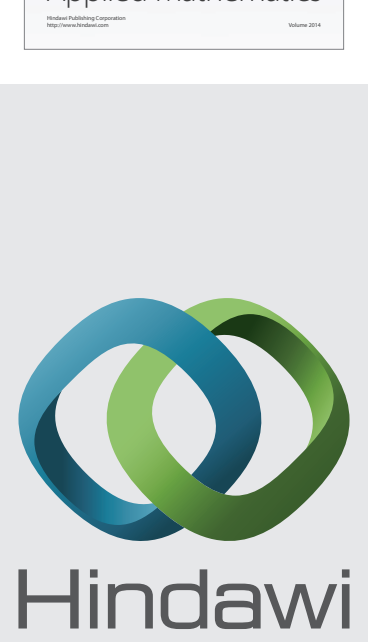

Submit your manuscripts at http://www.hindawi.com
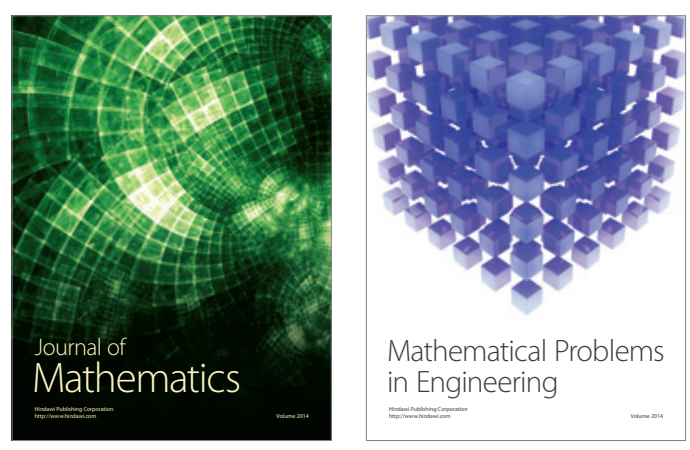

Mathematical Problems in Engineering
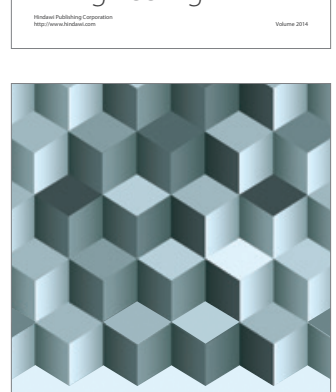

Journal of

Function Spaces
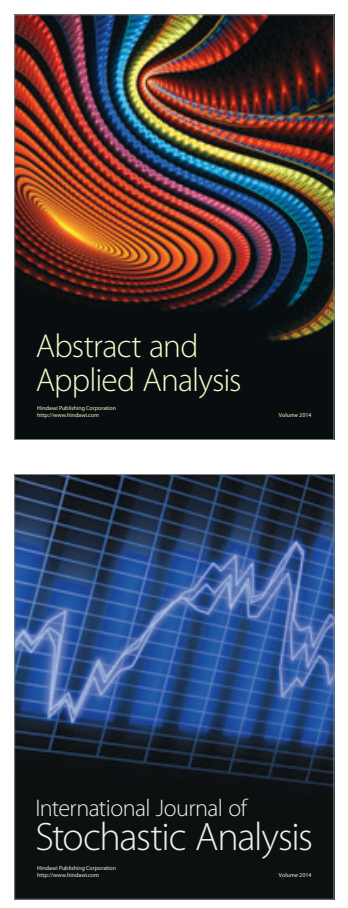

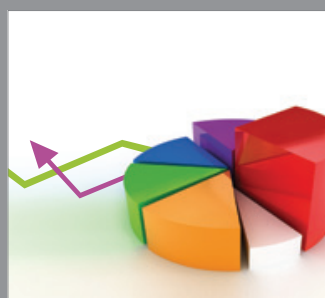

ournal of

Probability and Statistics

Promensencen
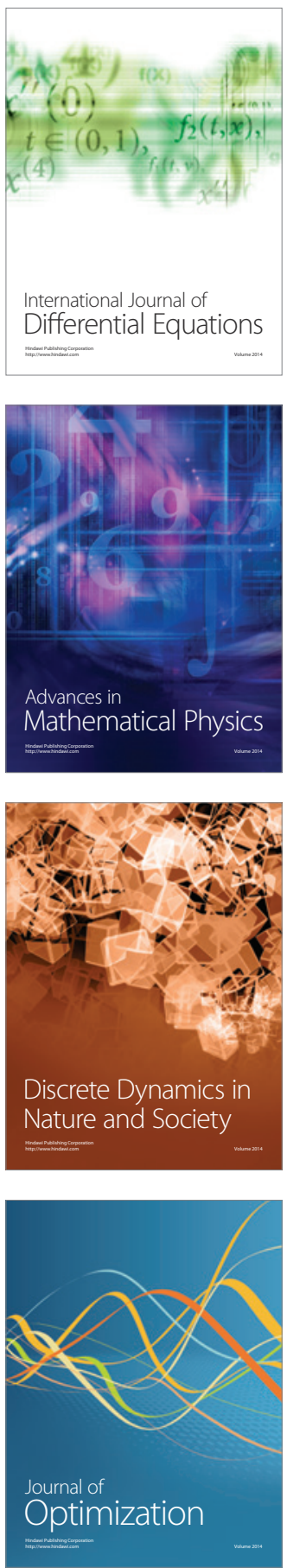